\title{
Moyamoya Syndrome with Recurrent Intraparenchymal Hemorrhage from Hemoglobin C Disease
}

Elizabeth Aradine*, Rachel Aubert and Ahmed Bayrlee

Virginia Commonwealth University, Richmond, VA, USA

\begin{abstract}
Moyamoya syndrome is an acquired idiopathic non-atherosclerotic vasculopathy that involves the terminal internal carotid artery and its branches. It is associated with numerous medical conditions such as hematologic abnormalities, autoimmune diseases, brain radiation, and tumors. These medical diseases cause chronic endothelial damage to cerebral vasculature resulting in both friability and stenosis of vessels, presenting as cerebral hemorrhage or infarction. There is an established link between moyamoya syndrome and hematologic diseases such as sickle cell and beta thalassemia. A few case reports of rare hemoglobin opathies have caused moyamoya syndrome; however, there are no reports of moyamoya due to hemoglobin $C$ disease. This case report presents a patient with known hemoglobin $C$ disease who presented with her second intracranial hemorrhage, found to have moyamoya syndrome.
\end{abstract}

Keywords: Moyamoya; Hemoglobin C disease; Intraparenchymal hemorrhage

\section{Introduction}

Moyamoya syndrome is an acquired idiopathic non-atherosclerotic vasculopathy that involves the terminal internal carotid artery and its branches. The syndrome is different from moyamoya disease as it is not a primary disease with associated genetic abnormality. Moyamoya syndrome has been associated with numerous medical diseases including but not limited to hematologic abnormalities, tumors, autoimmune diseases, or brain radiation. Both disease and syndrome usually have bilateral internal carotid and involvement; however can be unilateral. Angiography shows a characteristic "puff of smoke" appearance from collateralization of vasculature. Moyamoya syndrome presents more commonly as ischemic stroke in the pediatric population and hemorrhagic stroke in the adult population. Less common clinical presentations are epilepsy and movement disorders. Medical treatment consists of antiplatelet therapy to prevent thrombus formation. Surgical treatments include direct revascularization via anastomosis of the superficial temporal and middle cerebral artery which is more commonly used in children. Indirect revascularization consists of transposition of vasculature directly onto the cerebral cortex. It is unclear if surgical revascularization reduces the rate of hemorrhagic stroke.

\section{Case Presentation}

A 43-year-old right-handed female with type 1 von Willebrand's disease, homozygous hemoglobin $\mathrm{C}$ disease and prior left thalamic hemorrhagic stroke in 2014 without residual deficits originally presented to an outside hospital with generalized shaking activity for 5-9 min at home. She was found to have a left basal ganglia hemorrhage with intraventricular extension and was transferred to our hospital for neurosurgical intervention. $\mathrm{NIH}$ stroke scale was 29. Intracranial hemorrhage score was 3 and was intubated. Patient was not taking aspirin or anticoagulation prior to admission. Upon transfer to our hospital, her exam showed a presence of oculocephalic, corneal and gag reflexes. She did not withdraw to noxious stimulus in the right upper extremity but did grimace, localized in the left upper extremity, and withdrew to noxious stimulus in the lower extremities bilaterally but more briskly on the left than the right. She did not have upper motor neuron signs, clonus, or up toe responses. Patient had an extra ventricular drain placed on admission.
Laboratory studies showed microcytic anemia with baseline hemoglobin ranging from 8-9 throughout admission. Computed tomography angiogram (CTA) showed left intracranial and extracranial stenosis with extensive collateralization around Circle of Willis. Given the CTA findings, patient underwent a diagnostic cerebral angiogram which showed late stage moyamoya of the left anterior circulation (Figures 1 and 2).

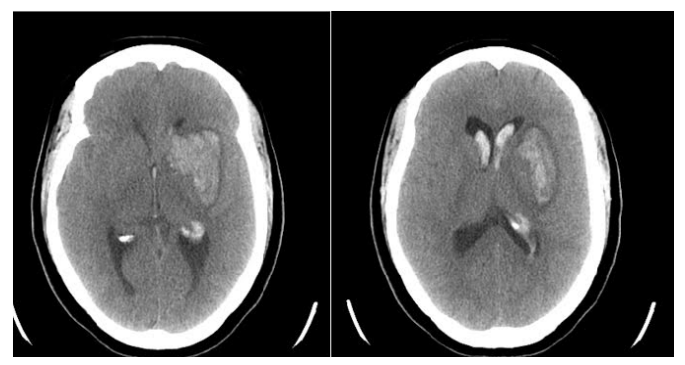

Figure 1: CT head.

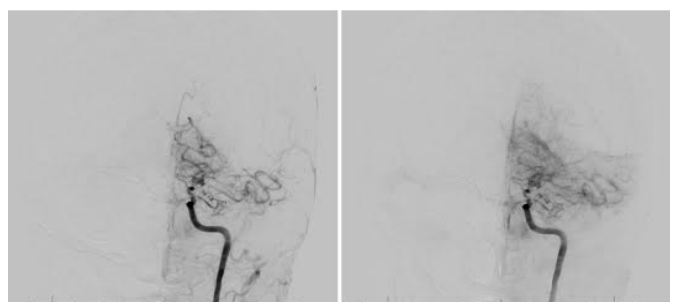

Figure 2: Angiography for our patient demonstrates the characteristic "puff of smoke" moyamoya pattern.

*Corresponding author: Elizabeth Aradine, Virginia Commonwealth University, Richmond, VA, Richmond, USA, Tel: 804-828-8593; Fax: 804-828-6499; E-mail: Elizabeth.m.aradine@vcuhealth.org

Received August 03, 2017; Accepted August 08, 2017; Published August 11, 2017

Citation: Aradine E, Aubert R, Bayrlee A (2017) Moyamoya Syndrome with Recurrent Intraparenchymal Hemorrhage from Hemoglobin C Disease. J Neurol Disord 5: 358. doi:10.4172/2329-6895.1000358

Copyright: @ 2017 Aradine E, et al. This is an open-access article distributed under the terms of the Creative Commons Attribution License, which permits unrestricted use, distribution, and reproduction in any medium, provided the original author and source are credited. 
Her hemoglobin $\mathrm{C}$ disease was associated with mild hemolytic anemia and mild splenomegaly, treated with daily folic acid and ferrous sulfate supplementation. On discharge, patient had right upper extremity and right hip flexor strength 4/5. 4 months after discharge, the patient underwent left encephalo-duro-arterio-synangiosis (EDAS). At her postoperative clinic visit, she had full strength in all muscle groups in her right upper and lower extremities. She is scheduled to have a 6 month postop diagnostic angiogram.

\section{Discussion}

Moyamoya syndrome is a non-atherosclerotic vasculopathy involving the cerebral vasculature, most commonly involving the internal carotid artery and its branches. The term "moya moya" is Japanese for "puff of smoke," referring to the characteristic angiographic appearance of vascular collateralization. Its pathogenesis is not well understood; however, basic fibroblast growth factor may play a role [1]. It is hypothesized that structurally abnormal red blood cells cause endothelial injury leading to endothelial proliferation resulting in vessel occlusion [1]. Histopathology shows intimal thickening from smooth muscle cell proliferation with medial fibrosis [1]. The medial fibrosis results in areas of discontinuity which then predisposes the vessel to hemodynamic stress [1]. In moyamoya vessels, it is noted that there is minimal or absent lipid deposition indicating a non-atherosclerotic process. Moyamoya has been associated with a variety of different hemoglobinopathies, genetic conditions that cause hemoglobin structural abnormalities resulting in abnormal red blood cells. The most widely known hemoglobinopathy is a mutation in hemoglobin $\mathrm{S}$, or sickle cell disease. Sickle cell disease has been a well-established risk for the development of moyamoya syndrome, most commonly presenting as ischemic stroke in the pediatric population. In the adult population, moyamoya syndrome presents as hemorrhagic stroke.

Hemoglobin C disease is the most common hemoglobinopathy and symptoms range from asymptomatic (if the patient is heterozygous) to mild hemolytic anemia and splenomegaly (if the patient is homozygous) [2]. While hemoglobin S mutation, or sickle cell disease, is less common, it has a higher morbidity and mortality with symptoms starting in childhood [3]. On peripheral blood smear, hemoglobin C red blood cells show hemoglobin crystals and target cells [4]. Diagnosis is made either by electrophoresis or liquid chromatography [5]. Current treatment for hemoglobin $\mathrm{C}$ disease is daily folic acid supplementation.

As far as we know, there have only been 4 reported cases of nonsickle cell hemoglobinopathies associated with moyamoya syndrome. A case of hemoglobin E [6], hemoglobin aleisha [7], hemoglobin Fairfax [8], and 2 cases associated with hemoglobin casper [9,10]. All above reported cases are in children. There is no reported non sickle cell disease hemoglobinopathies associated with moyamoya syndrome in adults. The above cases of hemoglobinopathies are rare and considered unstable with severe anemia requiring multiple blood transfusions. Hemoglobin $\mathrm{C}$ disease is considered to be a stable and more benign hemoglobinopathy as reported symptoms are less severe compared to the other hemoglobinopathies. Our patient with moyamoya syndrome from hemoglobin $\mathrm{C}$ disease proves otherwise and demonstrates that the long term risk of the same complications of a hemoglobinopathy but later in life. This could indicate that even patients with stable hemoglobinopathies show vessel changes, but manifest with these changes later in life.

There are no reports or studies of surgical treatment of moyamoya due to hemoglobin $\mathrm{C}$ disease; however, there have been reports of EDAS in children with moyamoya due to sickle cell disease. Surgical revascularization is successful in $75 \%$ to $90 \%$ of these patients at an average 4 year follow up with no new neurological complications [11]. Given that hemoglobin $C$ is a stable hemoglobinopathy with neurological complications presenting in the adult population rather than pediatric, it can be assumed that surgical revascularization is just as successful if not more as those with sickle cell disease.

\section{Conclusion}

Although hemoglobin C disease is considered a stable hemoglobinopathy with its only symptoms of mild hemolytic anemia and splenomegaly, the disease can damage cerebral vasculature similar to sickle cell disease but is a more insidious process. The vasculature is more friable hence the more common presentation of hemorrhage in adults versus stenosis in children. Patients who present with hemorrhagic stroke and have hematologic abnormalities should be evaluated for a hemoglobinopathy and moyamoya syndrome should be considered as an etiology.

\section{References}

\section{Singhal A (2015) Moyamoya disease.}

2. Powars DR, Hiti A, Ramicone E, Johnson C, Chan L (2002) Outcome in hemoglobin SC disease: A four-decade observational study of clinical, hematologic, and genetic factors. Am J Hematol 70: 206-15.

3. Strouse J (2016) Sickle cell disease. Handb Clin Neurol 138: 311-24.

4. Kraus AP, Diggs LW (1956) In vitro crystallization of hemoglobin occurring in citrated blood from patients with hemoglobin C. J Lab Clin Med 47: 700-705.

5. Smith EW, Krevans JR (1959) Clinical manifestations of hemoglobin C disorders. Bull Johns Hopkins Hosp 104: 17-43.

6. Parker TM, Ward LM, Johnston DL, Ventureya E, Klaassen RJ (2009) A case of Moyamoya syndrome and hemoglobin E/Beta-thalassemia. Pediatr Blood Cancer 52: 422-424.

7. Brockman K, Stolpe S, Fels C, Khan N, Kulozik AE, et al. (2005) Moyamoya syndrome associated with hemolytic anemia due to $\mathrm{Hb}$ alesha. J Pediatr Hematol Oncol 27: 436-446.

8. Marden FA, Putman CM, Grant JM, Greenberg J (2008) Moyamoya disease associated with hemoglobin Fairfax and beta-thalassemia. Pediatr Neurol 38: 130-132.

9. Haque A, Quint DJ, Castle VP, Leber SM (2015) Another rare unstable hemoglobinopathy: Hemoglobin casper/southampton associated with Moyamoya disease. Cerebrovasc Dis Extra 5: 52-54.

10. Delavari N, Strahle J, Maher CO (2013) Moyamoya syndrome associated with hemoglobin Southampton (Casper). Pediatr Neurosurg 49: 301-310.

11. Hankinson T, Bhoman LE, Heyer G, Licursi M, Ghatan S, et al. (2008) Surgical treatment of moyamoya syndrome in patients with sickle cell anemia: Outcome following encephaloduro-arteriosynangiosis. J Neurosurg: Peds 1: 211-216. 